\title{
HDAC6 promotes cell proliferation and confers resistance to gefitinib in lung adenocarcinoma
}

\author{
ZHIHAO WANG ${ }^{1,2}$, FANG TANG $^{1,2}$, PENGCHAO HU $^{3}$, YING WANG $^{3}$, JUN GONG $^{1,2}$, \\ SHAOXING SUN ${ }^{1,2}$ and CONGHUA XIE ${ }^{1,2}$ \\ ${ }^{1}$ Department of Radiation and Medical Oncology, and ${ }^{2}$ Hubei Key Laboratory of Tumor Biological Behaviors, \\ Zhongnan Hospital of Wuhan University; ${ }^{3}$ Department of Pathology and Pathophysiology, \\ School of Basic Medical Sciences, Wuhan University, Wuchang, Wuhan 430071, P.R. China
}

Received January 15, 2016; Accepted March 3, 2016

DOI: $10.3892 / o r .2016 .4811$

\begin{abstract}
Histone deacetylases (HDACs) are promising targets for cancer therapy, and first-generation HDAC inhibitors are currently in clinical trials for the treatment of cancer patients. HDAC6, which is a key regulator of many signaling pathways that are linked to cancer, has recently emerged as an attractive target for the treatment of cancer. In the present study, HDAC6 was found to be overexpressed in lung adenocarcinoma cell lines and was negatively correlated with the prognosis of patients with lung adenocarcinoma. Overexpression of HDAC6 promoted the proliferation of lung adenocarcinoma cells in a deacetylase activity-dependent manner. HDAC6 overexpression conferred resistance to gefitinib via the stabilization of epidermal growth factor receptor (EGFR). The inhibition of HDAC6 by CAY10603, a potent and selective inhibitor of HDAC6, inhibited the proliferation of lung adenocarcinoma cells and induced apoptosis. CAY10603 downregulated the levels of EGFR protein, which in turn inhibited activation of the EGFR signaling pathway. Moreover, CAY10603 synergized with gefitinib to induce apoptosis of the lung adenocarcinoma cell lines via the destabilization of EGFR. Taken together, our results suggest that the inhibition of HDAC6 may be a promising strategy for the treatment of lung adenocarcinoma.
\end{abstract}

Correspondence to: Dr Conghua Xie, Department of Radiation and Medical Oncology, Zhongnan Hospital of Wuhan University, 169 Donghu Road, Wuchang, Wuhan 430071, P.R. China

E-mail: chxie_65@whu.edu.cn

Abbreviations: SCLC, small cell lung cancer; NSCLC, non-small cell lung cancer; HDACs, histone deacetylases; EGFR, epidermal growth factor receptor; TKIs, tyrosine kinase inhibitors; ERK, extracellular signal-regulated kinase; Hsp90, heat shock protein 90; PARP, poly(ADP-ribose) polymerase; SIRT, silent mating type information regulation 2 homolog

Key words: HDAC6, lung adenocarcinoma, EGFR, CAY10603, gefitinib

\section{Introduction}

Lung cancer is the leading cause of cancer-related death worldwide and accounts for more than one million deaths per year (1). The morbidity of lung cancer has continued to increase worldwide, particularly in developing countries, which is partly due to air pollution. Lung cancer can be divided into two histological types: small cell lung cancer (SCLC) and non-small cell lung cancer (NSCLC). NSCLC can be further subdivided into: lung adenocarcinoma, large cell carcinoma and squamous cell carcinoma. These cancer subtypes have distinct morphologies and molecular profiles, and arise from distinct locations within the lung. Approximately $40 \%$ of lung cancers are adenocarcinomas. Despite advances in the diagnosis and treatment of lung adenocarcinoma, the 5-year overall survival rate of patients with lung adenocarcinoma is still extremely low (2). In recent years, great achievements have been made in our understanding of lung adenocarcinoma driver gene mutations and rearrangements, which play critical roles in tumor development and progression (3). Among them, somatic mutations in the gene that encodes the epidermal growth factor receptor (EGFR) and rearrangements that involve the gene that encodes anaplastic lymphoma kinase (ALK), are the most well-characterized examples (4,5). Large, prospective randomized trials have shown that molecularly selected patients with advanced lung adenocarcinoma can benefit from treatment with EGFR and ALK tyrosine kinase inhibitors (TKIs). However, despite the success of such agents in the treatment of genetically defined subsets of lung cancer, mutations such as those in EGFR and rearrangements within the ALK gene are identified in only a limited number of patients (6). Moreover, the majority of patients who initially respond to EGFR-TKI therapy develop acquired resistance within 6-12 months (5). Therefore, further research is needed to identify new therapeutic targets and tools for the treatment of lung adenocarcinoma.

Histone deacetylases (HDACs) are enzymes that modulate the acetylation status of histones and other important cellular proteins (7). Eighteen HDACs have been identified in humans, and these enzymes are divided into four classes, as follows, based on sequence phylogeny and function: class I (HDAC1, 2, 3 and 8), class II (HDAC4, 5, 6, 7, 9 and 10), class III 
(SIRT1-7) and class IV (HDAC11) (8). HDACs have been recognized as potentially useful therapeutic targets for a broad range of human disorders including cancer $(8,9)$. HDACs are considered to be among the most promising targets in drug development for cancer therapy. Two HDAC inhibitors, SAHA (vorinostat) and romidepsin (FK228), have been approved by the US Food and Drug Administration for the treatment of cutaneous T cell lymphoma (10,11). However, these inhibitors elicit profound side-effects since they target several HDAC isoforms $(12,13)$. Isoform-selective HDAC inhibitors may offer a therapeutic advantage due to minimal toxicity. Among the 18 HDACs, HDAC6 has recently sparked great interest due to its functional role in tumor progression. HDAC6 is a key regulator of many signaling pathways that have been linked to cancer $(14,15)$. A diverse set of HDAC6 substrates is involved in tumorigenesis, such as Hsp90, $\alpha$-tubulin and cortactin. Deacetylation of the Hsp90 core component by HDAC6 activates the chaperone activity of Hsp90 and stabilizes Hsp90-associated molecules including EGFR, Akt, c-Raf, FLT3 and mutant p53 (16). HDAC6 can promote cell migration through the deacetylation of $\alpha$-tubulin (17). HDAC6 has been shown to regulate the cell cycle, apoptosis, and metastasis, among other cellular processes $(18,19)$. However, unlike the inhibition of other HDACs, the inhibition of HDAC6 is not believed to be associated with severe toxicity, which makes HDAC6 a possible target for the treatment of cancer. However, few studies have been conducted in regards to the role of HDAC6 in lung adenocarcinoma.

In the present study, HDAC6 was found to be overexpressed in lung adenocarcinoma cell lines, and the overexpression of HDAC6 promoted lung adenocarcinoma proliferation and conferred resistance to gefitinib, a widely used EGFR-TKI, in a deacetylase activity-dependent manner. The inhibition of the deacetylase activity of HDAC6 by CAY10603 (20), a potent and selective HDAC6 inhibitor, inhibited the proliferation and induced th apoptosis of lung adenocarcinoma cell lines. CAY10603 synergized with gefitinib to induce the apoptosis of lung adenocarcinoma cells via the destabilization of EGFR. Our results suggest that inhibition of HDAC6 may be a promising strategy for the treatment of lung adenocarcinoma.

\section{Materials and methods}

Cell culture. The human lung adenocarcinoma cell lines A549, HCC827 and H1975 were obtained directly from the American Type Culture Collection (ATCC; Manassas, VA, USA). The cell lines were cultured in RPMI-1640 medium (HyClone, Logan, UT, USA) supplemented with $10 \%$ fetal bovine serum (FBS) and $1 \%$ penicillin/streptomycin.

Antibodies and reagents. CAY10603 and gefitinib were purchased from Selleck (Houston, TX, USA). The antiPARP (9542) and anti-BID (2002) antibodies were obtained from Cell Signaling Technology (Danvers, MA, USA). The anti-caspase 3 (EAP0893) antibody was purchased from Elabscience (Wuhan, Hubei, China). The anti-p53 (ab179477), anti-Bax (ab32503), anti-EGFR (ab52894) and anti-p-ERK (ab76299) antibodies were purchased from Abcam (Cambridge, MA, USA). The anti-HDAC6 (12834-1-AP), Bcl2 (12789-1-AP), Bcl-xL (10783-1-AP), Mcl1 (16225-1-AP), p21 (10355-1-AP) and ERK1/2 (16443-1-AP) antibodies were obtained from Proteintech (Chicago, IL, USA). The anti- $\beta$ actin mouse monoclonal antibody was purchased from Abgent (San Diego, CA, USA).

Cell proliferation and colony formation assays. In regards to the cell proliferation assay, cells that were cultured in 96-well plates were treated with the indicated compounds, and cell proliferation was measured at the indicated times by the Cell Counting Kit-8 (CCK-8) (Dojindo, Tokyo, Japan) assay. In regards to the colony formation assay, cells were seeded at 1,000/well into 6-well plates and were cultured over a 14-day period. Colonies were fixed in $4 \%$ paraformaldehyde, washed with $\mathrm{H}_{2} \mathrm{O}$ and stained with $0.1 \%$ crystal violet.

Annexin V assay of cell apoptosis. Cells were cultured in 6-well plates and were treated with the indicated compounds, trypsinized and collected. The collected cells were washed with PBS, resuspended in binding buffer, and stained with Annexin V-PE and 7-AAD for $15 \mathrm{~min}$ according to the manufacturer's protocol from Becton-Dickinson (San Jose, CA, USA). Fluorescence was estimated with a Becton-Dickinson flow cytometer.

Plasmids and transfection. The plasmids that express wild-type HDAC6 (HDAC6 WT) and deacetylase-deficient HDAC6 (HDAC6 MT) were kindly provided by Professor Jun Zhou of Nankai University $(21,22)$. The plasmids were transfected into cells with TurboFect DNA transfection reagent (Thermo Scientific, Waltham, MA, USA). Control siRNA and siRNAs targeting human HDAC6 were purchased from RiboBio (Guangzhou, China). siRNA was transfected into cells using Lipofectamine RNAiMAX from Invitrogen (Waltham, MA, USA).

Western blotting. Cells were lysed in lysis buffer (Beyotime, Jiangsu, China). The samples were electrophoresed by SDS-PAGE and transferred to polyvinylidene fluoride membranes (Roche). The membranes were blocked with 5\% dried skim milk and then incubated with the indicated primary antibody followed by incubation with the appropriate horseradish peroxidase (HRP)-conjugated secondary antibody (Proteintech). Finally, the bands were visualized with WesternBright ECL HRP substrate (Advansta, Menlo Park, CA, USA) and developed with Kodak film.

Statistical analysis. Statistical analysis was performed using the Student's t-test. Data are expressed as the mean \pm standard deviation (SD). $\mathrm{P}<0.05$ was considered to indicate a statistically significant difference.

\section{Results}

HDAC6 is overexpressed in lung adenocarcinoma and is negatively correlated with the prognosis of patients with lung adenocarcinoma. It has been reported that expression of HDAC6 protein is upregulated in several types of human cancers, but the role of HDAC6 in lung adenocarcinoma tumorigenesis is still unknown (19). To determine the expression level of HDAC6 protein in lung adenocarcinoma, 


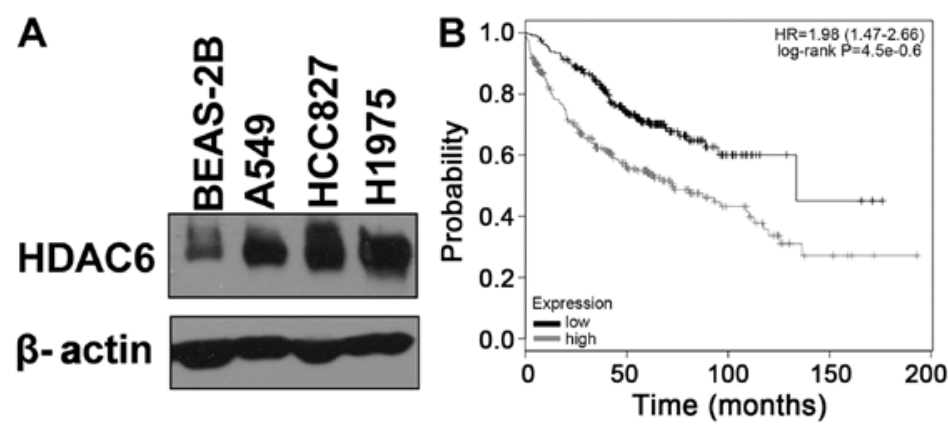

Figure 1. HDAC6 is upregulated in lung adenocarcinoma cell lines and is negatively correlated with the prognosis of patients with lung adenocarcinoma. (A) Western blot analysis of HDAC6 expression in three lung adenocarcinoma cell lines and the normal human lung cell line BEAS-2B. (B) The relationship between the expression of HDAC6 mRNA and the prognosis of patients with lung adenocarcinoma was analyzed using the Kaplan-Meier plotter database (version 2013) (23) (Kaplan-Meier plotter database website: http://kmplot.com/analysis/).
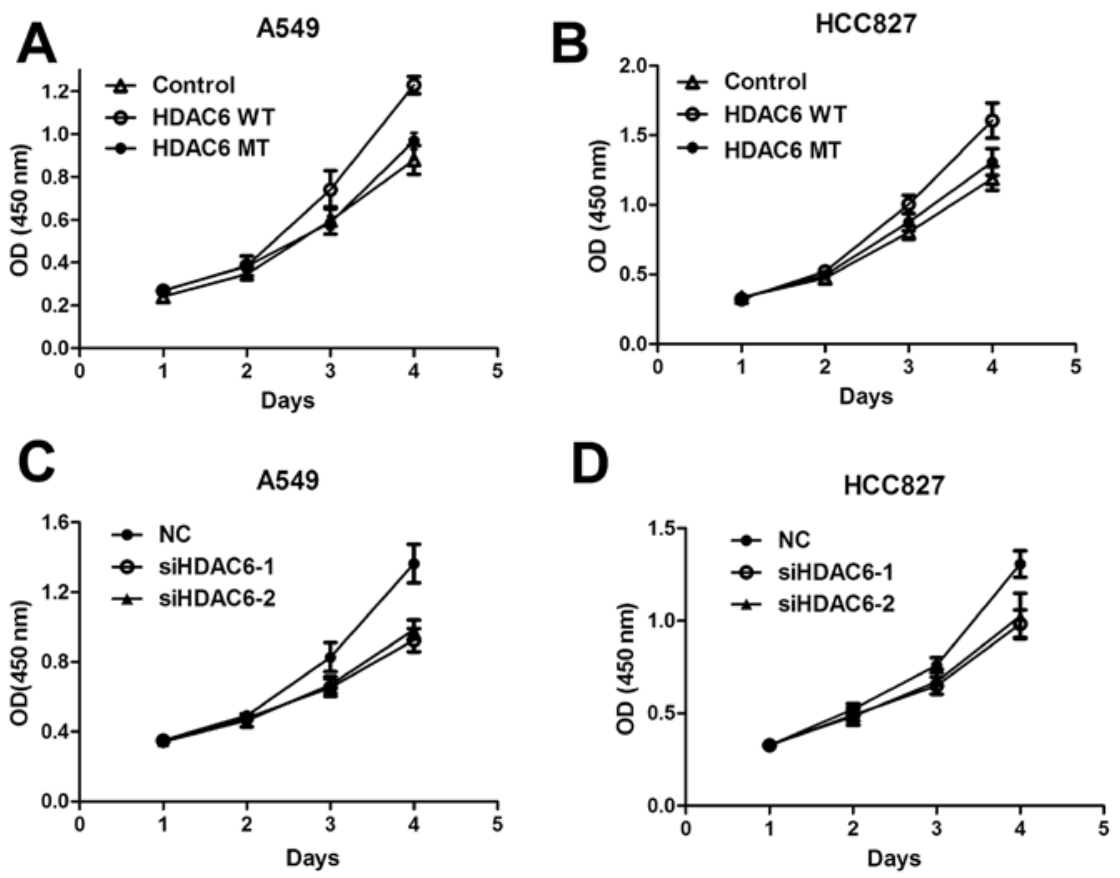

Figure 2. HDAC6 promotes the proliferation of lung adenocarcinoma cell lines. (A-D) Proliferation of A549 (A and C) and HCC827 (B and D) cells that were transfected with the indicated plasmids or siRNA was monitored by CCK-8 assay. NC, negative control.

we first compared the expression of HDAC6 between lung adenocarcinoma cell lines and a normal human lung cell line. Three lung adenocarcinoma cell lines (A549, HCC827 and H1975) and one normal human lung cell line (BEAS-2B) were tested. As shown in Fig. 1A, HDAC6 was overexpressed in the lung adenocarcinoma cell lines compared with the normal lung cell line. Moreover, following the search of an online Kaplan-Meier plotter database, and analysis of the prognostic value of biomarkers using transcriptomic data in NSCLC (23), we found that the expression of HDAC6 was negatively correlated with the prognosis of patients with lung adenocarcinoma $(\mathrm{P}<0.01)$ (Fig. 1B).

HDAC6 promotes the proliferation of lung adenocarcinoma cells in a deacetylase activity-dependent manner. Since HDAC6 is overexpressed in lung adenocarcinoma, we speculated that HDAC6 may regulate the proliferation of lung adenocarcinoma cells. To assess the effects of HDAC6 on cell proliferation and whether this is dependent on its deacetylase activity, A549 and HCC827 cells were transfected with wildtype HDAC6 (HDAC6 WT) and the catalytically inactive H216A/H611A mutant HDAC6 (HDAC6 MT) overexpression plasmids, and cell proliferation was measured by CCK-8 assay. We found a significant increase in the cell proliferation rate on day 4 when HDAC6 was upregulated (Fig. 2A and B). Moreover, the HDAC6 MT-transfected group showed nearly the same proliferation rate as the control group (Fig. 2A and B). Conversely, knockdown of HDAC6 led to impaired cell proliferation in both cell lines (Fig. 2C and D). These results indicate that HDAC6 promotes cell proliferation of lung adenocarcinoma in a deacetylase activity-dependent manner.

HDAC6 confers resistance to gefitinib-mediated killing of lung adenocarcinoma cells. Dramatic clinical responses to the selective EGFR-TKIs gefitinib and erlotinib have been observed in patients with advanced lung adenocarcinoma, 

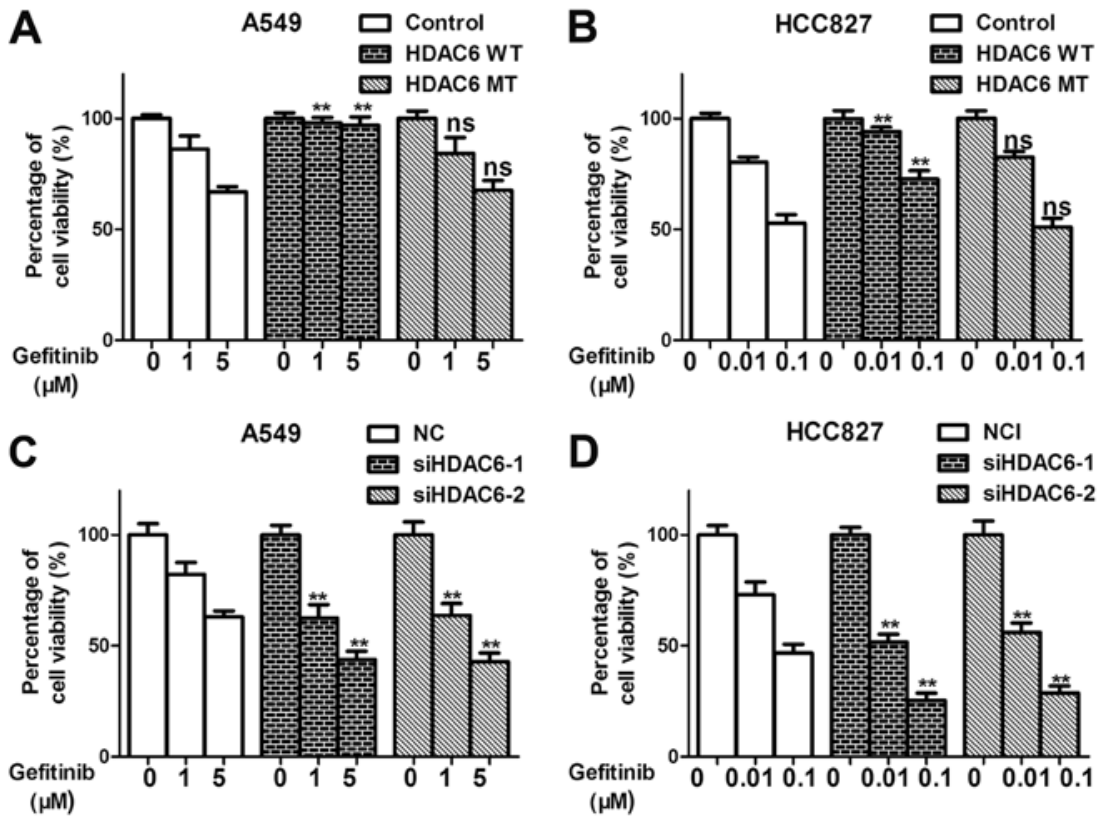

Figure 3. HDAC6 confers resistance to gefitinib-mediated cell proliferation inhibition. (A-D) A549 (A and C) and HCC827 (B and D) cells were transfected with the indicated plasmids or siRNA. Then, $36 \mathrm{~h}$ after transfection, the cells were treated with the indicated concentrations of gefitinib. After another $48 \mathrm{~h}$, cell viability was measured by CCK-8 assay. $\mathrm{NC}$, negative control. ${ }^{* *} \mathrm{P}<0.01$, t-test.

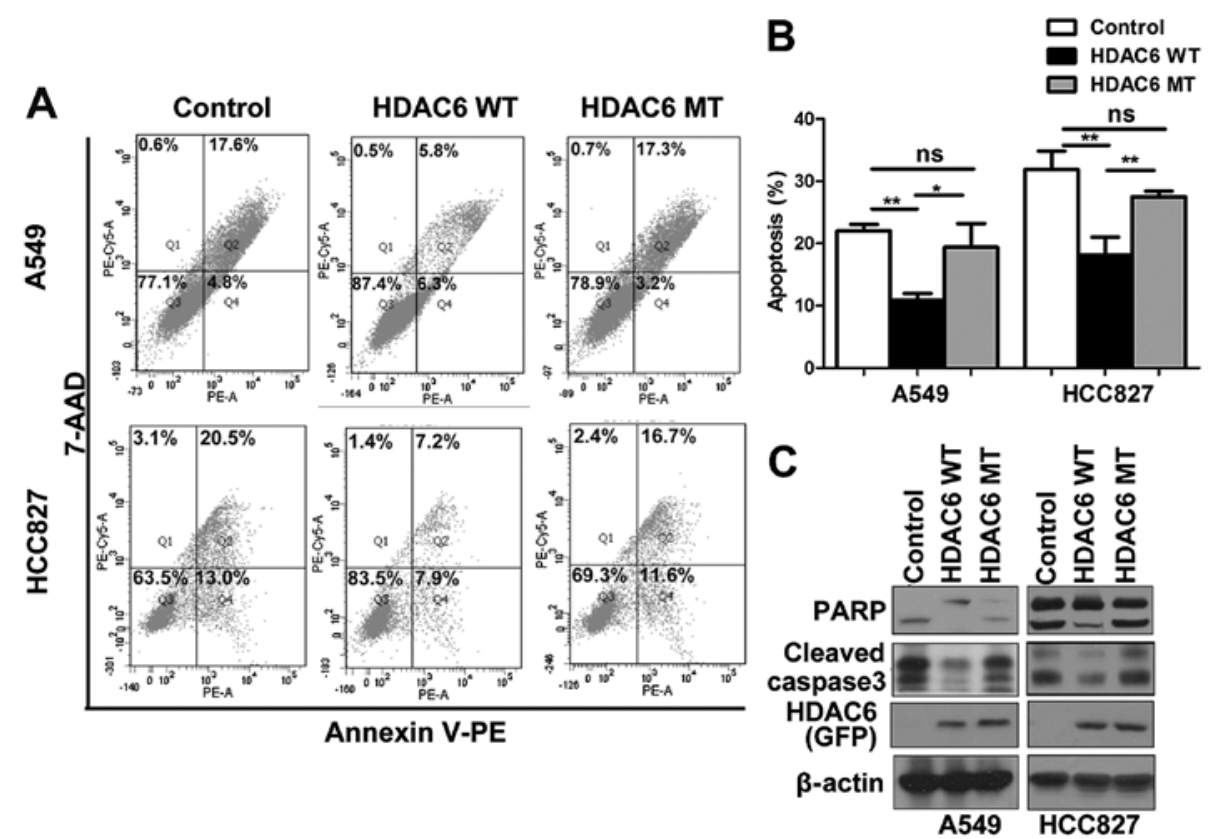

Figure 4. Overexpression of HDAC6 confers resistance to gefitinib-induced apoptosis. (A) A549 and HCC827 cells were transfected with wild-type HDAC6 or mutated HDAC6. Then, $36 \mathrm{~h}$ after transfection, the cells were treated with gefitinib; $5 \mu \mathrm{M}$ for A549 and $0.1 \mu \mathrm{M}$ for HCC 827 . After another $48 \mathrm{~h}$, cell apoptosis was detected by Annexin V staining (A) and western blotting (C). The percentage of apoptotic cells was derived from triplicate samples of Annexin V-positive cells as measured by flow cytometry $(\mathrm{B}){ }^{* *} \mathrm{P}<0.01$, t-test; ns, not significant.

particularly in those with tumors that harbor activating EGFR mutations (1). Gefitinib was the first small-molecule EGFR-TKI that received FDA approval in 2003. However, almost all tumors develop acquired resistance to these TKIs within 9-15 months (24). EGFR-TKIs are used to treat lung adenocarcinomas that carry activating EGFR mutations, whereas they exert only modest antitumor activity in lung adenocarcinomas that express wild-type EGFR. In the present study, we showed that the overexpression of HDAC6 remarkably impaired gefitinib efficiency with respect to the inhibition of cell proliferation of the lung adenocarcinoma cell lines A549 (EGFR WT) and HCC827 (EGFR DelE746A750) in a deacetylase activity-dependent manner (Fig. 3A and B). Knockdown of HDAC6 also sensitized the A549 and HCC827 cells to geftinib (Fig. 3C and D). Furthermore, by Annexin V staining and western blotting, the cleavage of PARP and caspase 3 was 


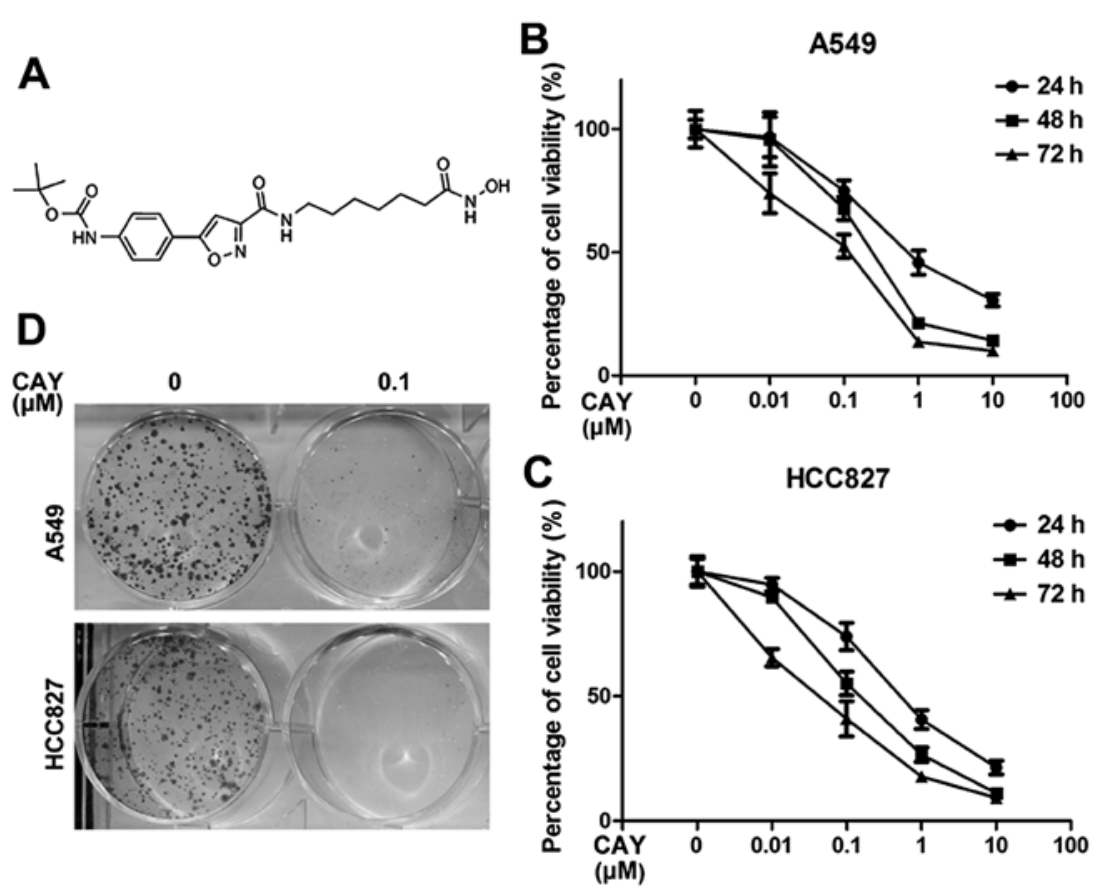

Figure 5. Inhibition of HDAC6 by CAY10603 inhibits the proliferation of lung adenocarcinoma cells. (A) The chemical structure of the selective HDAC6 inhibitor, CAY10603 (CAY). (B and C) Curves show the dose-dependent effect of CAY10603 on the viability of A549 (B) and HCC827 cells (C). (D) The effect of CAY10603 on cell clone formation was measured by plate clone formation assay.

A

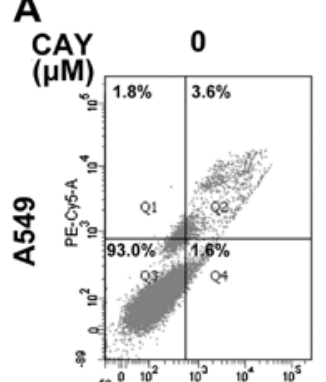

B
C

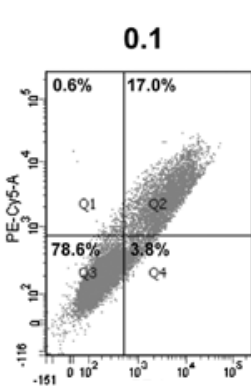

A549

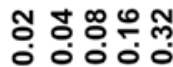

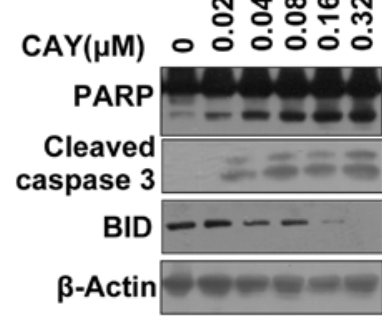

A549

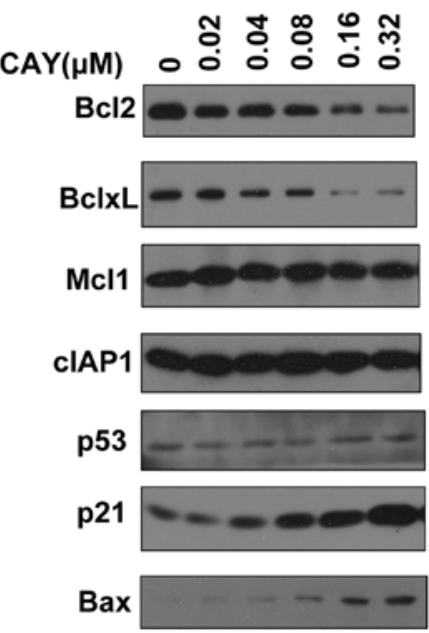

$\beta$-actin

Figure 6. CAY10603 induces apoptosis of lung adenocarcinoma cells. (A and B) A549 cells were treated with the indicated doses of CAY10603 (CAY). After $48 \mathrm{~h}$ of treatment, cell apoptosis was detected by Annexin V staining (A) and western blotting (B). (C) Western blot analysis of a panel of anti-apoptotic and pro-apoptotic proteins in samples treated with different doses of CAY10603.

detected and showed that HDAC6 overexpression conferred resistance to gefitinib-induced apoptosis, which was dependent on the deacetylase activity of HDAC6 (Fig. 4A-C). Our results suggest that the overexpression of HDAC6 may be an intrinsic mechanism that confers resistance to EGFR-TKIs.

Inhibition of HDAC6 deacetylase activity by CAY10603, a selective HDAC6 inhibitor, inhibits the proliferation of lung adenocarcinoma cells. The above data indicated that HDAC6 plays a relevant role in the control of lung adenocarcinoma cell proliferation. To further assess this possibility, we tested the effect of HDAC6 inhibition on lung adenocarcinoma cell lines using a potent and selective inhibitor, CAY10603 (chemical structure shown in Fig. 5A). We assessed the growth inhibition in response to CAY10603 treatment in two human lung adenocarcinoma cell lines: A549 and HCC827. A dose-dependent decrease in cell proliferation was observed for both cell lines tested (Fig. 5B and C). The same conclusion was drawn from 
A

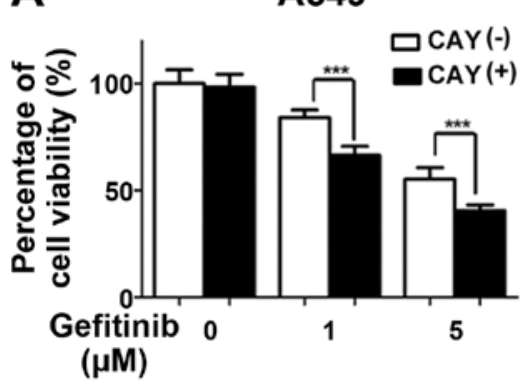

B

CAY
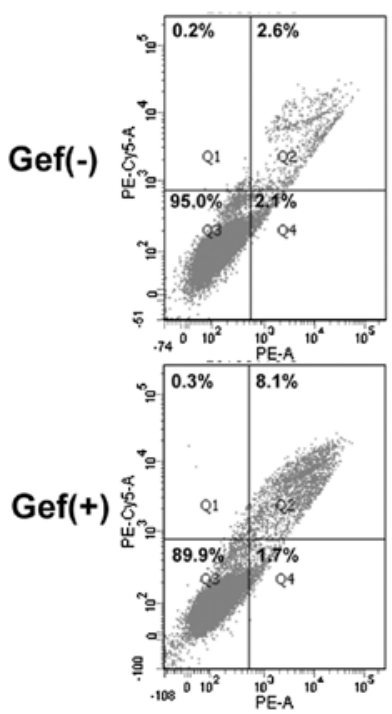

A549
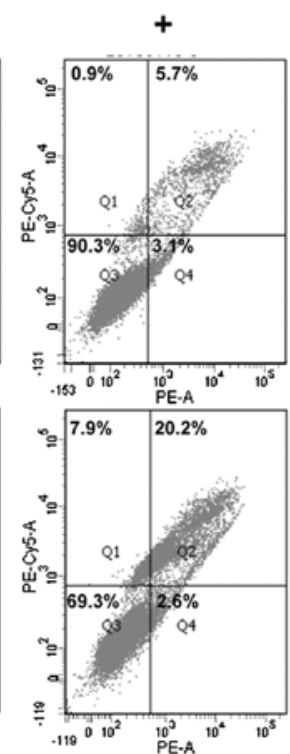

C
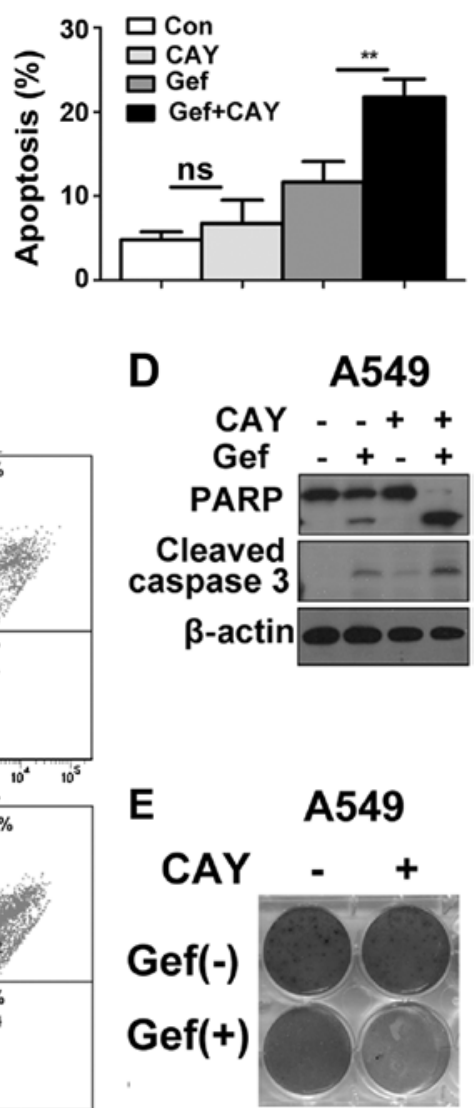

Figure 7. Inhibition of HDAC6 by CAY10603 synergizes with gefitinib to decrease the survival of A549 cells. (A) A549 cells were treated with the given doses of gefitinib (Gef) and $0.01 \mu \mathrm{M}$ CAY10603 (CAY). Cell viability was measured by CCK-8 assay. (B-D) A549 cells were treated with $1 \mu \mathrm{M}$ gefitinib and $0.01 \mu \mathrm{M}$ CAY 10603 for $48 \mathrm{~h}$. Cell apoptosis was detected by Annexin V staining (B) and western blotting (D). The percentage of apoptotic cells was derived from triplicate samples of Annexin V-positive cells as measured by flow cytometry (C). (E) A549 cells were plated into 6-well plates and were treated with the indicated chemicals. Long-term survival was visualized after 7 days by crystal violet staining. One of three independent experiments is shown. ${ }^{* *} \mathrm{P}<0.01, \mathrm{t}-\mathrm{test}$

the results of the clone formation assay. We noted a significant decrease in clone numbers of the lung adenocarcinoma cell lines after CAY10603 treatment (Fig. 5D).

Inhibition of HDAC6 by CAY10603 notably induces apoptosis of lung adenocarcinoma cells. Given that CAY10603 inhibits the proliferation of lung adenocarcinoma cell lines, we investigated its effect on apoptosis. CAY10603 clearly induced apoptosis in the lung adenocarcinoma cell line A549 (Fig. 6A). We determined the effects of CAY10603 on the activation of caspases and Bid proteins as well as its effect on PARP cleavage, which serves as a marker of apoptosis. Western blot analysis demonstrated that PARP, Bid and caspase 3 were cleaved following CAY10603 treatment in the A549 cells (Fig. 6B). Protein levels of the apoptosis-related proteins were also detected (Fig. 6C). The inhibition of HDAC6 by CAY10603 increased the levels of pro-apoptotic proteins such as BAX and p21 (Fig. 6C). CAY10603 also led to decreases in the levels of anti-apoptotic proteins including $\mathrm{Bcl} 2$ and Bcl-xL (Fig. 6C).

Inhibition of HDAC6 synergizes with gefitinib to induce apoptosis in lung adenocarcinoma cell lines, partly through the destabilization of EGFR and inactivation of the EGFR pathway. Since HDAC6 overexpression confers resistance to gefitinib, we speculated that the inhibition of HDAC6 may contribute to an increase in the efficiency of gefitinib with respect to lung adenocarcinoma cells. To examine whether a cooperative effect exists between CAY10603 and gefitinib in the chemotherapeutic treatment of lung adenocarcinoma, we treated A549 cells with CAY10603 and gefitinib either alone or in combination. In agreement with our hypothesis, the co-treatment of CAY10603 and gefitinib significantly reduced the cell viability of the gefitinib-resistant cell line A549 (Fig. 7A). The rate of apoptosis was also increased after the co-treatment of these two drugs, as evidenced by increases in the number of Annexin V-positive cells (Fig. 7B and C). An increase in apoptosis by this combination drug treatment was further evidenced by the cleavage of PARP and caspase 3 (Fig. 7D). We also observed that the combination of CAY10603 and gefitinib remarkably inhibited the clonogenic survival of the A549 cells (Fig. 7E). These data suggest that the combination treatment of CAY10603 and gefitinib displayed a significant synergistic therapeutic effect in lung adenocarcinoma. Next, we aimed to ascertain the mechanism by which CAY10603 contributes to the gefitinib-induced cell 

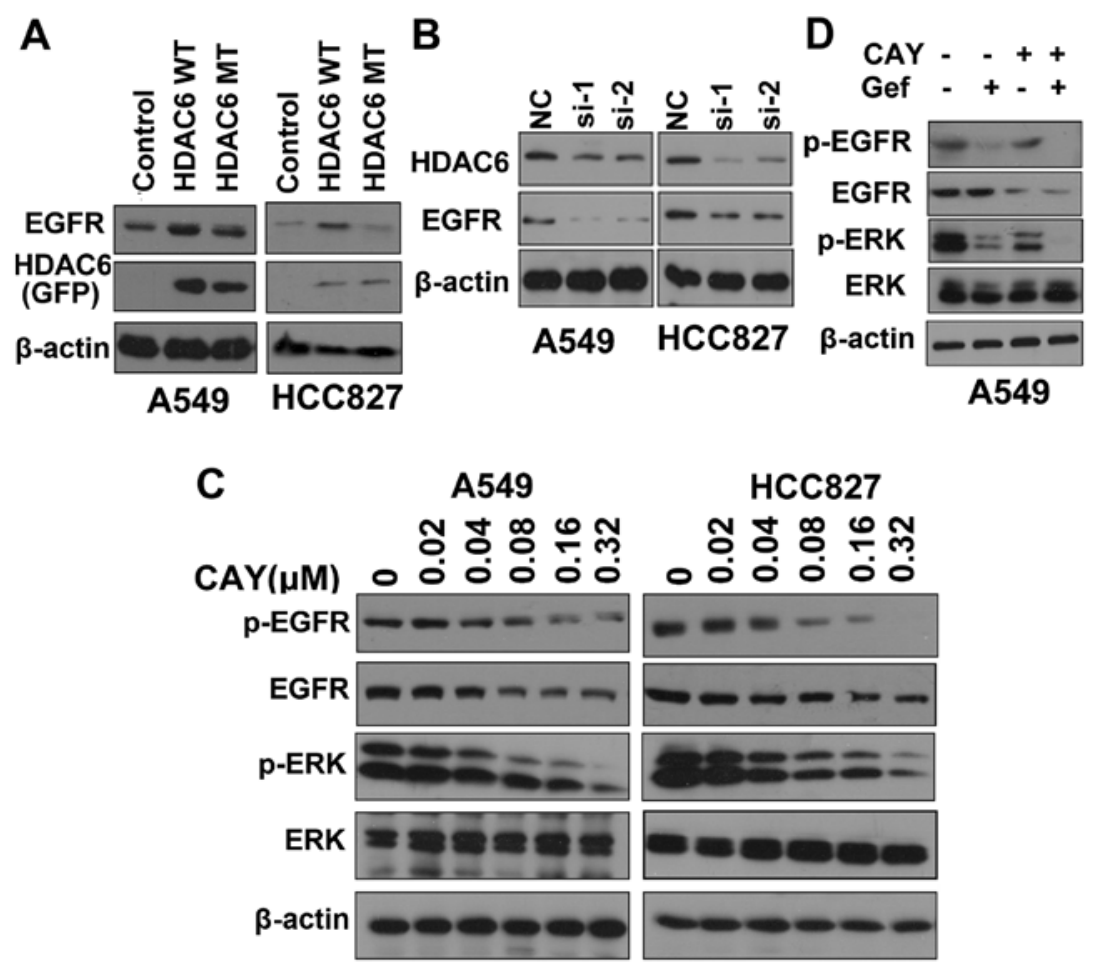

Figure 8. HDAC6 stabilizes EGFR in a deacetylase activity-dependent manner and inhibition of HDAC6 inactivates the EGFR pathway. (A) A549 and HCC827 cells were transfected with wild-type HDAC6 or with mutated HDAC6. Then, $36 \mathrm{~h}$ after transfection, the cells were harvested for preparation of whole-cell protein lysates and subsequent western blot analysis. (B) A549 and HCC827 cells were transfected with negative control (NC) or HDAC6-specific siRNA. Then, $72 \mathrm{~h}$ after transfection, the cells were harvested for preparation of whole-cell protein lysates and subsequent western blot analysis. (C) Cells were treated with the indicated doses of CAY10603 (CAY). After $72 \mathrm{~h}$ of treatment, the cells were harvested for preparation of whole-cell protein lysates and subsequent western blot analysis. (D) A549 cells were plated in 6-well cell culture plates, and the next day, they were treated with $1 \mu \mathrm{M}$ gefitinib (Gef) and $0.01 \mu \mathrm{M}$ CAY10603, the cells were harvested for preparation of whole-cell protein lysates and subsequent western blot analysis.

death of lung adenocarcinoma cells. HDAC6 has been shown to be an important regulator of EGFR endocytic trafficking and degradation $(25,26)$. To test whether HDAC6 regulates EGFR stability in lung adenocarcinoma cells, the cells were transfected with control or HDAC6 overexpression plasmids for $48 \mathrm{~h}$, and then the cells were lysed and EGFR protein levels were detected by western blotting. We found that overexpression of HDAC6 in lung adenocarcinoma cell lines stabilized EGFR in a deacetylase activity-dependent manner (Fig. 8A). Conversely, knockdown of HDAC6 led to destabilization of EGFR (Fig. 8B). Thus, the inhibition of HDAC6 may also destabilize EGFR and inhibit activation of the EGFR pathway. CAY10603 treatment destabilized EGFR and inhibited activation of the EGFR pathway in the A549 and HCC 827 cells. ERK, a well-known EGFR target, was selected as a symbol of EGFR activation (Fig. 8C). We also found that the activation of ERK was inhibited by the combination of CAY10603 and gefitinib compared with the administration of either inhibitor alone (Fig. 8D). Taken together, our results showed that inhibition of HDAC6 synergized with gefitinib to induce apoptosis in lung adenocarcinoma via destabilization of EGFR and inactivation of the EGFR pathway.

\section{Discussion}

HDACs are enzymes that are involved in the regulation of the acetylation of histone and non-histone proteins. Protein acetylation has emerged as an important post-translational modification that regulates multiple cellular functions, including chromatin remodeling and transcriptional regulation, microtubule dynamics, metabolism, autophagy and apoptosis $(7,12)$. HDACs are considered to be among the most promising targets for cancer therapy, and first-generation HDAC inhibitors are currently being tested in phase I/II clinical trials (9). SAHA and romidepsin have already been approved by the US FDA for the treatment of cutaneous T cell lymphoma. As a cytoplasmic HDAC, HDAC6 has been shown to deacetylate a diverse set of substrates that are involved in tumorigenesis, including Hsp90, $\alpha$-tubulin and EGFR $(16,25,26)$. HDAC6 has also been implicated in the regulation of many cancer-associated cellular events and signaling pathways, which makes it an attractive target for cancer therapy (27). HDAC6 has been shown to contribute to the tumorigenesis of several human cancers, such as medulloblastoma, cholangiocarcinoma and prostate cancer (27-29). HDAC6 inhibitors are being tested both in preclinical settings and clinical trials as an anticancer agent (ClinicalTrials.gov Identifier: NCT01323751, NCT02091063, NCT02632071 and NCT02635061). However, the role of HDAC6 in lung adenocarcinoma has not been well characterized. In the present study, we demonstrated that HDAC6 is overexpressed in lung adenocarcinoma cell lines and promotes the oncogenesis of lung adenocarcinoma. HDAC6 also stabilized EGFR in lung adenocarcinoma cells. The inhibition of HDAC6 by CAY10603, a potent and selective HDAC6 inhibitor, induced apoptosis and impaired the proliferation of lung 
adenocarcinoma cells. Moreover, the inhibition of HDAC6 synergized with EGFR-TKIs to induce the apoptosis of lung adenocarcinoma cells via the suppression of EGFR pathway activation.

Although mounting evidence predicts a therapeutic benefit for HDAC inhibitors, we still face significant challenges in their clinical application. Broad spectrum HDAC inhibitors block multiple HDAC isoforms, particularly HDAC1 and HDAC3 $(12,13)$. Although HDAC inhibitors have demonstrated significant antitumor effects, the profound side-effects associated with their use cannot be ignored. However, unlike the inhibition of other HDACs, the inhibition of HDAC6 is not believed to be associated with severe toxicity (30). Moreover, HDAC6 knockout in mice does not lead to embryonic lethality (31). Given that HDAC6 plays an important role in lung adenocarcinoma, the inhibition of HDAC6 may induce apoptosis in lung adenocarcinoma cells without the accompaniment of as many side-effects as broad spectrum HDAC inhibitors. Further studies that compare the antitumor efficiency and safety of HDAC6 inhibitors with broad spectrum HDAC inhibitors are needed, particularly in animal models.

EGFR plays a critical role in the control of cellular proliferation, differentiation and survival of lung adenocarcinoma cells. Activating mutations in EGFR such as exon 19 deletions and the L858R substitution in exon 21 are important markers of response to EGFR-TKI therapy in lung adenocarcinoma (24). Acquired resistance to EGFR-TKIs is a major problem that continues to puzzle clinicians since the majority of patients who initially respond to therapy develop acquired resistance within 6-12 months. Several mechanisms that may be responsible for this acquired resistance have been identified, including a secondary T790M mutation in EGFR, c-Met amplification and transformation to SCLC (5). In addition to these well characterized mechanisms, further elucidation of novel mechanisms of acquired drug resistance is essential to overcome the problem of EGFR-TKI resistance. Furthermore, patients without EGFR mutations still manifest acquired resistance to EGFR inhibitors. Therefore, it remains urgent to explore additional mechanisms of acquired resistance to EGFR inhibitors. As mentioned above, the overexpression of HDAC6 stabilizes EGFR, while the inhibition of HDAC6 destabilizes EGFR. Here, we showed that overexpression of HDAC6 may partly account for the intrinsic resistance to EGFR-TKIs due to the activation of the EGFR pathway.

The kinase-independent role of EGFR has attracted increased attention in recent years. Zhang et al found that the survival of cancer cells can be maintained by EGFR independently of its kinase activity through the prevention of autophagic cell death of cancer cells (32). Thus, EGFR can still promote the survival of cancer cells when kinase activity is blocked by EGFR-TKIs. Acceleration of the degradation of EGFR may solve this problem, and the inhibition of HDAC6 can both destabilize EGFR and inhibit activation of the EGFR pathway. This may partly explain the synergistic effects of CAY10603 and gefitinib.

In summary, we report that HDAC6 is upregulated in lung adenocarcinoma and that inhibition of HDAC6 impairs the proliferation of lung adenocarcinoma cells. Moreover, the HDAC6 inhibitor CAY10603 synergizes with gefitinib to induce apoptosis in lung adenocarcinoma cells, which occurs partly through the destabilization of EGFR and inactivation of the EGFR pathway. Therefore, the inhibition of HDAC6 may be a potential strategy for treating lung adenocarcinoma and overcoming resistance to EGFR-TKIs.

\section{Acknowledgements}

We acknowledge Professor Jun Zhou of the Nankai University for kindly providing the HDAC6 overexpression plasmids. The present study was supported by the Natural Sciences Foundation of Hubei Province (no. 2013CFA006).

\section{References}

1. Pao W and Girard N: New driver mutations in non-small-cell lung cancer. Lancet Oncol 12: 175-180, 2011.

2. Ettinger DS, Wood DE, Akerley W, Bazhenova LA, Borghaei H, Camidge DR, Cheney RT, Chirieac LR, D'Amico TA, Demmy TL, et al; National comprehensive cancer network: Non-Small Cell Lung Cancer, Version 6.2015. J Natl Compr Canc Netw 13: 515-524, 2015.

3. Pao W and Chmielecki J: Rational, biologically based treatment of EGFR-mutant non-small-cell lung cancer. Nat Rev Cancer 10: 760-774, 2010.

4. Popper HH, Ryska A, Tímár J and Olszewski W: Molecular testing in lung cancer in the era of precision medicine. Transl Lung Cancer Res 3: 291-300, 2014.

5. Politi K and Herbst RS: Lung cancer in the era of precision medicine. Clin Cancer Res 21: 2213-2220, 2015.

6. Reck M,Heigener DF, Mok T, Soria JC and Rabe KF: Management of non-small-cell lung cancer: Recent developments. Lancet 382: 709-719, 2013.

7. Minucci S and Pelicci PG: Histone deacetylase inhibitors and the promise of epigenetic (and more) treatments for cancer. Nat Rev Cancer 6: 38-51, 2006.

8. Haberland M, Montgomery RL and Olson EN: The many roles of histone deacetylases in development and physiology: Implications for disease and therapy. Nat Rev Genet 10: 32-42, 2009.

9. Falkenberg KJ and Johnstone RW: Histone deacetylases and their inhibitors in cancer, neurological diseases and immune disorders. Nat Rev Drug Discov 13: 673-691, 2014

10. Duvic M, Olsen EA, Breneman D, Pacheco TR, Parker S, Vonderheid EC, Abuav R, Ricker JL, Rizvi S, Chen C, et al: Evaluation of the long-term tolerability and clinical benefit of vorinostat in patients with advanced cutaneous T-cell lymphoma. Clin Lymphoma Myeloma 9: 412-416, 2009.

11. Marks PA and Breslow R: Dimethyl sulfoxide to vorinostat: Development of this histone deacetylase inhibitor as an anticancer drug. Nat Biotechnol 25: 84-90, 2007.

12. Bruserud Ø, Stapnes C, Ersvaer E, Gjertsen BT and Ryningen A: Histone deacetylase inhibitors in cancer treatment: A review of the clinical toxicity and the modulation of gene expression in cancer cell. Curr Pharm Biotechnol 8: 388-400, 2007.

13. Marsoni S, Damia G and Camboni G: A work in progress: The clinical development of histone deacetylase inhibitors. Epigenetics 3: 164-171, 2008.

14. Kaliszczak M, Trousil S, Åberg O, Perumal M, Nguyen QD and Aboagye EO: A novel small molecule hydroxamate preferentially inhibits HDAC6 activity and tumour growth. Br J Cancer 108: 342-350, 2013.

15. Namdar M, Perez G, Ngo L and Marks PA: Selective inhibition of histone deacetylase 6 (HDAC6) induces DNA damage and sensitizes transformed cells to anticancer agents. Proc Natl Acad Sci USA 107: 20003-20008, 2010.

16. Krämer OH, Mahboobi S and Sellmer A: Drugging the HDAC6HSP90 interplay in malignanT cells. Trends Pharmacol Sci 35: 501-509, 2014.

17. Zhang Y, Li N, Caron C, Matthias G, Hess D, Khochbin S and Matthias P: HDAC-6 interacts with and deacetylates tubulin and microtubules in vivo. EMBO J 22: 1168-1179, 2003. 
18. Park SJ, Kim JK, Bae HJ, Eun JW, Shen Q, Kim HS, Shin WC, Yang HD, Lee EK, You JS, et al: HDAC6 sustains growth stimulation by prolonging the activation of EGF receptor through the inhibition of rabaptin-5-mediated early endosome fusion in gastric cancer. Cancer Lett 354: 97-106, 2014.

19. Aldana-Masangkay GI and Sakamoto KM. The role of HDAC6 in cancer. J Biomed Biotechnol 2011: 875824, 2011.

20. Kozikowski AP, Tapadar S, Luchini DN, Kim KH and Billadeau DD: Use of the nitrile oxide cycloaddition (NOC) reaction for molecular probe generation: A new class of enzyme selective histone deacetylase inhibitors (HDACIs) showing picomolar activity at HDAC6. J Med Chem 51: 4370-4373, 2008.

21. Yang Y, Ran J, Liu M, Li D, Li Y, Shi X, Meng D, Pan J, Ou G, Aneja R, et al: CYLD mediates ciliogenesis in multiple organs by deubiquitinating Cep70 and inactivating HDAC6. Cell Res 24: 1342-1353, 2014

22. Ran J, Yang Y, Li D, Liu M and Zhou J: Deacetylation of $\alpha$-tubulin and cortactin is required for HDAC6 to trigger ciliary disassembly. Sci Rep 5: 12917, 2015.

23. Győrffy B, Surowiak P, Budczies J and Lánczky A: Online survival analysis software to assess the prognostic value of biomarkers using transcriptomic data in non-small-cell lung cancer. PLoS One 8: e82241, 2013

24. Yu HA, Arcila ME, Rekhtman N, Sima CS, Zakowski MF, Pao W, Kris MG, Miller VA, Ladanyi M and Riely GJ: Analysis of tumor specimens at the time of acquired resistance to EGFR-TKI therapy in 155 patients with EGFR-mutant lung cancers. Clin Cancer Res 19: 2240-2247, 2013.

25. Deribe YL, Wild P, Chandrashaker A, Curak J, Schmidt MH, Kalaidzidis Y, Milutinovic N, Kratchmarova I, Buerkle L, Fetchko MJ, et al: Regulation of epidermal growth factor receptor trafficking by lysine deacetylase HDAC6. Sci Signal 2: ra84, 2009.
26. Gao YS, Hubbert CC and Yao TP: The microtubule-associated histone deacetylase 6 (HDAC6) regulates epidermal growth factor receptor (EGFR) endocytic trafficking and degradation. J Biol Chem 285: 11219-11226, 2010.

27. Gradilone SA, Radtke BN, Bogert PS, Huang BQ, Gajdos GB and LaRusso NF: HDAC6 inhibition restores ciliary expression and decreases tumor growth. Cancer Res 73: 2259-2270, 2013.

28. Dhanyamraju PK, Holz PS, Finkernagel F, Fendrich V and Lauth M: Histone deacetylase 6 represents a novel drug target in the oncogenic Hedgehog signaling pathway. Mol Cancer Ther 14: 727-739, 2015.

29. Ai J, Wang Y, Dar JA, Liu J, Liu L, Nelson JB and Wang Z: HDAC6 regulates androgen receptor hypersensitivity and nuclear localization via modulating Hsp90 acetylation in castrationresistant prostate cancer. Mol Endocrinol 23: 1963-1972, 2009.

30. Haggarty SJ, Koeller KM, Wong JC, Grozinger CM and Schreiber SL: Domain-selective small-molecule inhibitor of histone deacetylase 6 (HDAC6)-mediated tubulin deacetylation. Proc Natl Acad Sci USA 100: 4389-4394, 2003.

31. Kaluza D, Kroll J, Gesierich S, Yao TP, Boon RA, Hergenreider E, Tjwa M, Rössig L, Seto E, Augustin HG, et al: Class IIb HDAC6 regulates endothelial cell migration and angiogenesis by deacetylation of cortactin. EMBO J 30: 4142-4156, 2011.

32. Weihua Z, Tsan R, Huang WC, Wu Q, Chiu CH, Fidler IJ and Hung MC: Survival of cancer cells is maintained by EGFR independent of its kinase activity. Cancer Cell 13: 385-393, 2008. 